\title{
Potentiale der Kreislaufwirtschaft zur Reduktion des Ausstoßes von Treibhausgasen
}

\author{
Lorenzo Rieg, Anna Meyer und Hanno Bertignoll \\ Resources Innovation Center, Montanuniversität Leoben, Leoben, Österreich \\ Eingegangen 8. Februar 2019; angenommen 15. Februar 2019; online publiziert 28. Februar 2019
}

Zusammenfassung: Die Abhängigkeit von fossilen Brennstoffen, deren Verbrennung nach wie vor den größten Teil der Treibhausgasemissionen der Menschheit ausmacht, ist hauptsächlicher Treiber des globalen Klimawandels. Die Kreislaufwirtschaft, welche die Wiederverwendung und Reparatur von Produkten und das Recycling von Materialien beinhaltet, kann einen entscheidenden Beitrag leisten, um den $\mathrm{CO}_{2}$-Fußabdruck von Materialien und Produkten zu verringern. In diesem Artikel werden Potentiale zur Reduzierung von Treibhausgasemissionen in den Materialsystemen Stahl, Aluminium und Kunststoff aufgezeigt und analysiert.

Schlüsselwörter: Kreislaufwirtschaft, Rohstoffe, Klimawandel

Potentials of the Circular Economy for the Reduction of Greenhouse Gas Emissions

Abstract: The dependence on fossil fuels, which are still responsible for the majority of the greenhouse gas emissions of humanity, is a strong driver for global climate change. Circular economy, which includes the reuse and repair of products and the recycling of materials, can contribute significantly to a reduction of the $\mathrm{CO}_{2}$ footprint of materials and products. In this paper, potentials for the reduction of greenhouse gas emissions, mainly in the material systems of steel, aluminium and plastics, are shown and analyzed.

Keywords: Circular economy, Raw materials, Climate change

\section{Einleitung}

Nach wie vor ist die Abhängigkeit von fossilen Brennstoffen das Hauptproblem im Bereich der Mitigation des globalen Klimawandels. Über die Hälfte der weltweiten Treibhausgasemissionen stammt aus der Verbrennung fossiler Energieträger [1]. Es ist daher wenig verwunderlich, dass sich ein großer Teil der Bemühungen um eine Begrenzung des Klimawandels, wie sie etwa 2015 im Rahmen des Übereinkommens von Paris beschlossen wurden [2], auf die Erhöhung der Energieeffizienz und erneuerbare Energien konzentriert.

Doch auch andere Ansätze zur Reduktion des Treibhausgasausstoßes rücken immer mehr in den Vordergrund. So auch die Kreislaufwirtschaft, welche neben den klaren Vorteilen im Bereich der Ressourcenverfügbarkeit und des Umweltschutzes auch ein hohes Potential zur Klimawandelmitigation mitbringt. Dies wurde beispielsweise bereits $2015 \mathrm{im}$ Aktionsplan der Europäischen Kommission [3] festgehalten, der zusätzlich auch zeigt, dass die Kreislaufwirtschaft nicht nur zu einer signifikanten Einsparung von Treibhausgasen, gerade im industriellen Sektor, sondern auch zum Erhalt der Wettbewerbsfähigkeit der Europäischen Industrie beitragen kann [4]. Durch die Bemühungen der Europäischen Kommission, etwa mit dem "Abfallpaket" [5], wird die Kreislaufwirtschaft auch seitens der Gesetzgeber in Europa stärker gefördert und gefordert.

\section{Potentiale zur Einsparung von Treibhaus- gasen durch Kreislaufwirtschaft}

Die Produktion und der Verbrauch von Materialien und Produkten ist für einen bedeutenden Teil des Ausstoßes von Treibhausgasen verantwortlich, weswegen die Wiederverwendung oder Reparatur von Produkten beziehungsweise das Recycling von Materialien den $\mathrm{CO}_{2}$-Fußabdruck von Produkten und Stoffen deutlich reduzieren kann.

So könnten Berechnungen zufolge alleine in den Sektoren Transport, Bau und Nahrungsmittelerzeugung bis zum Jahr 2050 über $60 \%$ der $\mathrm{CO}_{2}$-Emissionen durch die kon- 
sequente Umsetzung der Kreislaufwirtschaft eingespart werden [6, 7]. Bezogen auf die vier Materialsysteme mit den höchsten Emissionen, Stahl, Aluminium, Zement und Kunststoff, könnten bis zum Jahr 2100 etwa $30 \%$ der $\mathrm{CO}_{2-}$ Emissionen eingespart werden [8].

Hinzu kommt, dass die Kreislaufwirtschaft als systemtransformativer Prozess gesehen werden kann, bei dem nicht nur bestehende Wirtschaftsmodelle Abfall liefern, für den neue und bessere Lösungen zu Wieder- und Weiterverwendung gefunden werden sollen. Vielmehr geht es auch um den Aufbau von globalen Netzwerken, um die geografische Verteilung von Ressourcen anzusprechen. Ziel ist eine Vernetzung von Produkten, Komponenten und Material, um entlang der Wertschöpfungskette zusammenzuarbeiten und Informationslücken zu schließen. Sind diese Lücken geschlossen, wird es auch leichter, Design-for-Recycling-Ansätze umzusetzen. Des Weiteren gehören neue Wirtschaftsformen, die das Teilen und Mieten anstatt Besitzen vermarkten, zu den Treibern der Kreislaufwirtschaft, da durch geteilte Nutzung weniger $\mathrm{CO}_{2}$-Emissionen entstehen als durch klassische Besitzformen [9].

\subsection{Stahl}

Obwohl die Produktion von Stahl aktuell für einen großen Teil der industriellen Emissionen der Treibhausgase verantwortlich ist, ist Stahl auch heutzutage bereits ein verhältnismäßig zirkuläres Material [8]. Das Potential zur Reduktion von Emissionen ist noch nicht ausgeschöpft, es sind hier allerdings starke Verbesserungen in den nächsten Jahren und Dekaden zu erwarten.

Bereits in der Vergangenheit konnte durch Forschung und Entwicklung, neue Technologien und Verbesserungen im Design von Produkten sowohl die Menge an Rohmaterial als auch an Energie für die Herstellung von Stahlprodukten reduziert werden. In Zukunft sind hier weitere Verbesserungen zu erwarten, etwa durch eine weitere Verbreitung von Hochleistungsstählen oder durch optimiertes Design von Produkten [10]. Als Beispiel kann der Leichtbau von Automobilen genannt werden, durch den Energie eingespart werden kann.

Stahl ist aufgrund seiner Materialeigenschaften, etwa der hohen Haltbarkeit, sehr gut zur Wiederverwendung oder Wiederaufbereitung geeignet. Im Vergleich zu anderen Materialien findet dies auch bereits in verschiedenen Bereichen statt. Beispielsweise wird von Wiederverwendungsraten zwischen 5 und $10 \%$ für Stahl aus Gebäuden in Großbritannien [11] und in Australien [12] berichtet. Durch verbessertes Design von Produkten ist mit einer deutlichen Erhöhung von Wiederverwendungsraten von Stahl zu rechnen, wobei aktuell sowohl ökonomische als auch regulatorische Hürden dem entgegenstehen [11].

Stahl ist ein Material mit hoher Recyclingquote [10], nicht nur aufgrund des verhältnismäßig hohen Wertes von Stahlschrott, sondern auch aufgrund der leichten Rückgewinnung aus fast jedem Abfallstrom. Projektionen zufolge soll die Recyclingquote bis 2050 weiter ansteigen, was zu einer deutlichen Reduktion des $\mathrm{CO}_{2}$-Ausstoßes führen wird, da bei der Produktion von Primärstahl aktuell etwa die 10-fa- chen Emissionen im Vergleich zu Sekundärstahl verursacht werden [8].

Abgesehen von dem Potential durch die Kreislaufwirtschaft wird sich der $\mathrm{CO}_{2}$-Fußabdruck von Stahl in Zukunft durch neue Technologien, etwa im Bereich der Wasserstoffmetallurgie, deutlich verringern lassen. Hierbei könnte Strom aus erneuerbaren Energieträgern gewonnen werden, um mit Elektrolyse Wasserstoff herzustellen, der dann verwendet wird, um Stahl aus Eisenerz zu gewinnen. In Österreich könnte so eine Menge von ca. zwölf Millionen Tonnen $\mathrm{CO}_{2}$ eingespart werden [13].

\subsection{Aluminium}

Aluminium ist dank der Kombination aus hoher spezifischer Festigkeit und geringem Gewicht aus vielen Anwendungen, gerade im Leichtbau, nicht wegzudenken. Allerdings ist die Produktion von Primäraluminium bekanntlich sehr energieintensiv, was zu einem hohen $\mathrm{CO}_{2}$-Fußabdruck führt. Aluminium zeigt aber auch hohes Potential für den Klimaschutz in Bezug auf seine hohe Zirkularität. Die Produktion von Sekundäraluminium benötigt nur etwa $5 \%$ der Energie im Vergleich zur Primärproduktion [8]. Entscheidend ist daher eine weitere Steigerung der Recyclingrate, obwohl aktuell bereits ca. $75 \%$ des Aluminiums nach Ende der Lebensdauer der entsprechenden Produkte gesammelt wird [14].

Zusätzlich wird sich der $\mathrm{CO}_{2}$-Fußabdruck von Aluminium durch klimafreundlichere Energiequellen in Zukunft verbessern

\subsection{Kunststoff}

Im Gegensatz zu Stahl und Aluminium, bei denen der Bedarf in Europa nur leicht ansteigt, wird in Zukunft deutlich mehr Kunststoff benötigt werden. Dies wird auch zu einer Zunahme an Treibhausgasemissionen führen, zumal die Recyclingraten von Kunststoffen nach wie vor sehr gering sind. Während aktuell die Energierückgewinnung durch das Verbrennen von Kunststoff nach der Nutzung durch die breite Verwendung von fossilen Rohstoffen zur Energiegewinnung kaum ins Gewicht fällt, wird in einer Gesellschaft, in welcher ein Großteil der Energie aus erneuerbaren Quellen gewonnen wird, die Energierückgewinnung aus Kunststoffen im heutigen Stil entscheidend zu den Treibhausgasemissionen beitragen.

Insgesamt bietet das Materialsystem der Kunststoffe dadurch ein sehr hohes Potential zur Reduzierung von $\mathrm{CO}_{2-}$ Emissionen. So wird aktuell in der EU etwa $10 \%$ des Bedarfs an Kunststoff aus Sekundärkunststoffen gedeckt [8]. Technisch wären hier über $50 \%$ möglich, allerdings ist der Europäische Markt für Sekundärkunststoffe kaum entwickelt.

\section{Herausforderungen}

Den unbestrittenen wirtschaftlichen und Klimaschutzpotentialen der Kreislaufwirtschaft stehen zahlreiche Herausforderungen gegenüber, welche in den nächsten Jahren be- 
wältigt werden müssen, um den möglichen Beitrag zum Klimaschutz auch zu leisten.

Für alle Materialsysteme werden bessere Sammlung, die Vermeidung von "Downcycling", also der Umwandlung in Materialien oder Produkte von geringerem Wert, und ein funktionierender Markt für Sekundärrohstoffe entscheidende Herausforderungen der näheren Zukunft darstellen. Zudem muss zunehmend schon in der Produktentwicklung und im Produktdesign der gesamte Wertschöpfungskreislauf berücksichtigt werden. Das beinhaltet beispielsweise die Entwicklung von Szenarien für die Wiederverwendung, die Wiederaufbereitung und das Recycling, etwa um die Reinheit von Materialien zu gewährleisten, bereits bei der Entwicklung von Produkten.

\subsection{Stahl}

Im Gegensatz zu Kunststoffen ist die Verfügbarkeit von Sekundärmaterial bei Stahl bereits relativ hoch [13]. Zudem wird sie in der Zukunft voraussichtlich weiter steigen und sich somit der Nachfrage stark annähern [8]. Ein Problem hierbei ist allerdings die Vielfalt an Stählen und die oft strikten Anforderungen an die Qualität und Materialeigenschaften. Dies führt aktuell dazu, dass sekundärer Stahl häufig für Anwendungen mit niedrigeren Ansprüchen an die Materialqualität verwendet wird [15].

Ein weiteres Problem stellt die Verunreinigung von Stahl mit Kupfer, bzw. in geringerem Maß auch mit anderen Elementen wie etwa Nickel, dar [16]. Bereits geringe Anteile von Kupfer im Stahl können die Qualität drastisch verringern, so wird Stahl bereits ab einem Kupfergehalt von lediglich 0,1\% für zahlreiche Anwendungen unbrauchbar [17]. Da Kupfer aus dem Stahl praktisch nicht mehr zu gewinnen ist, führt die Verunreinigung zu einer dauerhaften Abwertung des Materials. Kupfer gelangt meist während des Recyclingprozesses in den Stahl, da viele Komponenten oder Produkte beide Materialien enthalten.

\subsection{Aluminium}

Durch den hohen Energiebedarf der Produktion von Primäraluminium kann durch erhöhte Recyclingraten ein signifikanter Beitrag zur Reduzierung von $\mathrm{CO}_{2}$-Emissionen erreicht werden. Hierzu muss vor allem noch mehr Aluminium gesammelt und somit für Wiederverwendung, Wiederaufbereitung und Recycling zur Verfügung gestellt werden.

Weiterhin wird die Verringerung des Downcyclings von Aluminium in Legierungen mit höheren Gehalten an anderen Metallen eine wichtige Rolle spielen. Diese Legierungen können häufig nicht mehr geschmiedet werden, sondern finden in der Gießerei Verwendung. Aktuell geschieht das häufig im Automobilbereich, allerdings kann hier mit einer Zunahme von Elektroautos ein verringerter Bedarf erwartet werden, da zahlreiche Teile im Motor, welche aktuell aus gegossenem Aluminium hergestellt werden, in Elektroautos nicht vorhanden sind.

\subsection{Kunststoff}

Ein Ziel sollte die Erhöhung des Anteils an Sekundärmaterial sein, wobei hier verschiedene Probleme zu bewältigen sind. Um hochqualitatives Rezyklat aus Kunststoffen herstellen zu können, müssten verschiedene Kunststoffe idealerweise getrennt gesammelt und getrennten Abfallströmen zugeführt werden.

So machen verschiedene Additive, wie beispielsweise Farbstoffe oder Stabilisatoren, sekundäre Kunststoffe häufig ungeeignet für die Nutzung in neuen Produkten. Auch Kontamination durch andere Stoffe, etwa durch die Inhalte von Kunststoffverpackungen, ist ein häufiges Problem, das ein Recycling verhindert oder zumindest erschwert. Ein weiteres Hindernis sind auch die hohen Kosten, die mit der Aufarbeitung und Aufbereitung von Sekundärkunststoffen verbunden sind.

\section{Fazit}

Die kurz vorgestellten Potentiale zur Reduktion von Treibhausgasemissionen in verschiedenen Materialsystemen zeigen die Bedeutung des Übergangs zur Kreislaufwirtschaft aus der Sicht des Klimaschutzes. Die Herausforderungen, welche in der Zukunft bewältigt werden müssen, sind technischer, aber auch gesellschaftlicher Natur und bedürfen daher Engagement aus verschiedensten Teilen der Gesellschaft. Ein gesteigertes Bewusstsein der Problematik, aber auch Willen zur Implementierung möglicher Lösungen wird dazu führen, dass die notwendigen Änderungen zeitnah stattfinden.

Funding. Open access funding provided by Montanuniversität Leoben.

Open Access Dieser Artikel wird unter der Creative Commons Namensnennung 4.0 International Lizenz (http://creativecommons.org/licenses/ by/4.0/deed.de) veröffentlicht, welche die Nutzung, Vervielfältigung,

Bearbeitung, Verbreitung und Wiedergabe in jeglichem Medium und Format erlaubt, sofern Sie den/die ursprünglichen Autor(en) und die Quelle ordnungsgemäß nennen, einen Link zur Creative Commons Lizenz beifügen und angeben, ob Änderungen vorgenommen wurden.

Hinweis des Verlags. Der Verlag bleibt in Hinblick auf geografische Zuordnungen und Gebietsbezeichnungen in veröffentlichten Karten und Institutsadressen neutral.

\section{Literatur}

1. Allen, R.M.; Barros, V.R.; Broome, J.; Cramer, W.; Christ, R.; Church, J.A.; Clarke, L.; Dahe, Q.; Dasgupta, P.; Dubash, N.K.; Edenhofer, O.; Elgizouli, I.; Field, C.B.; Forster, P.; Friedlingstein, P.; Fuglestvedt, J.; Gomez-Echeverri, L.; Hallegatte, S.; Hegerl, G.; Howden, M.; Jiang, K.; Jimenez Cisneros, B.; Kattsov, V.; Lee, H.; Mach, K.J.; Marotzke, J.; Mastrandrea, M.D.; Meyer, L.; Minx, J.; Mulugetta, Y.; O’Brien, K.; Oppenheimer, M.; Pachauri, R.K.; Pereira, J.J.; Pichs-Madruga, R.; Plattner, G.-K.; Pörtner, H.-O.; Power, S.B.; Preston, B.; Ravindranath, N.H.; Reisinger, A.; Riahi, K.; Rusticucci, M.; Scholes, R.; Seyboth, K.; Sokona, Y.; Stavins, R.; Stocker, T.F.; Tschakert, P.; van Vuuren, D.; van Ypersele, J.-P.; Blanco, G.; Eby, M.; Edmonds, J.; Fleurbaey, M.; Gerlagh, R.; Kartha, S.; Kunreuther, H.; Rogelj, J.; Schaeffer, M.; Sedláček, J.; Sims, R.; Ürge-Vorsatz, D.; Victor, D.; Yohe, G.: IPCC fifth assessment synthesis report-Climate Change 2014 synthesis report, IPCC, 2014 
2. Paris Agreement of the parties to the United Nations Framework Convention on Climate Change, 04.11.2016, document C.N.92.2016

3. European Commission: Closing the loop-An EU action plan for the Circular Economy. Brussels, 2015

4. Bijleveld, M.; Bergsma, G.; Nusselder, S.: The circular economy as a key instrument for reducing climate change, Delft: CE Delft, 2016, Publication code: $16.2 \mathrm{H} 81.51$

5. Kadenbach, K.: Weniger Müll, mehr Wiederverwertung und Recycling: Das neue EU-Abfallrecht auf dem Weg in die Kreislaufwirtschaft. Europainfo, 2 (2018), p. 6

6. Ellen MacArthur Foundation and McKinsey Center for Business and Environment: Growth Within: A circular economy vision for a competitive Europe, 1. publ. June 25, 2015, https://www. ellenmacarthurfoundation.org/publications/growth-within-acircular-economy-vision-for-a-competitive-europe (Accessed February 9, 2019)

7. Deloitte Sustainability: Circular economy potential for climate change mitigation, 2016, https://www2.deloitte.com/de/de/pages/ risk/articles/circular-economy-potential-for-climate-changemitigation.html (Accessed February 9, 2019)

8. Material Economics: The Circular Economy-A Powerful Force For Climate Mitigation, 2018, http://materialeconomics.com/ (Accessed February 9, 2019)

9. World Economic Forum: Towards the Circular Economy: Accelerating the scale-up across global supply chains, http://reports. weforum.org/toward-the-circular-economy-accelerating-the-scaleup-across-global-supply-chains/?doing_wp_cron=1549809249. 2815299034118652343750 (Accessed February 9, 2019)
10. World Steel Association: Steel in the circular economy-A life cycle perspective, http://worldsteel.org, 2015 (Accessed February 8, 2019)

11. Tingley, D.D.; Cooper, S.; Cullen, J: Understanding and overcoming the barriers to structural steel reuse, a UK perspective, Journal of Cleaner Production, 148 (2017), p. 642-652

12. Hardie, M.; Khan, S.; Miller, G.: Waste minimisation in office refurbishment projects: An Australian perspective, The Open Waste Management Journal, 4 (2011), p. 21-27

13. Bundesministerium für Verkehr, Wirtschaft und Technologie (2017): Auch die Stahlschmelze soll grün werden. https://infothek.bmvit.gv. at/auch-die-stahlschmelze-soll-gruener-werden/ (Accessed January 29, 2019)

14. Bertram, M.; Ramkumar, S.; Rechberger. H.; Rombach, G.; Bayliss, C.; Martchek, K.J.; Müller, D.B.; Liu, G.: A regionally-linked, dynamic material flow modelling tool for rolled, extruded and cast aluminium products. Resources, Conservation and Recycling, 125 (2017), p. 48-69

15. Willeke, R.: World Steel Recycling in Figures 2013-2017. BIR - Bureau of International Recycling, Brussels, 2018

16. Ohno, H.; Matsubae, K.; Nakajima, K.; Nakamura, S.; Nagasaka, T. Unintentional Flow of Alloying Elements in Steel during Recycling of End-of-Life Vehicles, Journal of Industrial Ecology, 18 (2014), 2, p. 391-401

17. Daehn, K.E.; Cabrera Serrenho, A.; Allwood, J.M.: How Will Copper Contamination Constrain Future Global Steel Recycling? Environmental Science and Technology, 51 (2017), 11, p.6599-6606 